\title{
Interculturalidad en salud: reflexiones a partir de una experiencia indígena en la Amazonía peruana
}

\section{Cynthia Giovanna Cárdenas}

Grupo de estudios sobre pueblos indígenas, Universidad Federal Belem do Pará cintiacardenas@gmail.com

\section{María Amalia Pesantes}

Universidad Peruana Cayetano Heredia maria.amalia.pesantes@gmail.com

\section{Alfredo Rodríguez}

Universidad Nacional Mayor de San Marcos

alfredo.alroto2002@gmail.com

\section{RESUMEN}

Este artículo presenta y analiza las principales características de la propuesta de interculturalidad desarrollada por una organización indigena amazónica del Perú en la formación de jóvenes indigenas como enfermeros técnicos en salud intercultural. A través de este programa de formación, mostramos cómo la Asociación Interétnica de Desarrollo de la Selva Peruana (Aidesep) se apropia del concepto de interculturalidad, lo resignifica y reconstruye en la práctica. A partir de documentos internos, publicaciones institucionales, testimonios de egresados, dirigentes y miembros del equipo técnico del Programa de Salud Indígena de Aidesep, analizamos la construcción e implementación del concepto de interculturalidad. Este artículo muestra la forma en que una organización indigena se vuelve un actor proponente de la interculturalidad, construyendo una respuesta indígena para la formación de profesionales técnicos, preparados para brindar atención en salud culturalmente apropiada a la población indigena. La propuesta de formación de enfermeros técnicos en salud intercultural demuestra que es posible que la 
interculturalidad crítica pase del discurso a la práctica cuando los pueblos indigenas construyen propuestas interculturales propias.

Palabras clave: interculturalidad, pueblos indigenas, salud intercultural, Amazonía, Perú.

\title{
Interculturality in health: reflections from an indigenous experience in the Peruvian Amazon
}

\author{
ABSTRACT
}

This article examines the main characteristics of the proposal on interculturality developed by an indigenous Amazonian organization of Peru for the training of indigenous youth as nurse technicians in intercultural health. It shows how the Interethnic Association for the Development of the Peruvian Jungle (Aidesep) appropriates the concept of interculturality, reconfigures and reconstructs it. Based on internal documents, institutional publications, testimonies of graduates of the intercultural health program, leaders and members of the technical team of the Indigenous Health Program of AIDESEP, we analyze the construction and implementation of the concept of interculturality. We also examin the way in which an indigenous organization becomes a proponent of interculturality, building an indigenous response for the training of health professionals prepared to provide culturally appropriate health services to the indigenous population. The proposal for the training of nurse technicians in intercultural health shows that it is possible for critical interculturality to move from discourse to practice when indigenous peoples develop their own intercultural proposals.

Keywords: interculturality, indigenous peoples, intercultural health, Amazonia, Peru. 


\section{INTRODUCCIÓN}

La interculturalidad entendida críticamente aún no existe, es algo por construir. Catherine Walsh (2009, p. 4)

En América Latina, se han construido alrededor de la interculturalidad discursos y propuestas que se han plasmado en experiencias sobre todo educativas y de salud. Estas experiencias han sido impulsadas desde el Estado, la academia, los organismos no gubernamentales y las organizaciones indígenas, entre otras. A diferencia del contexto europeo, donde la interculturalidad es un enfoque usado sobre todo para mejorar la relación con las minorías étnicas, en Latinoamérica la interculturalidad ha estado vinculada con la atención de la diversidad cultural y la problemática indígena (López, 2001). Las organizaciones indígenas promueven la interculturalidad como una estrategia para producir cambios en las estructuras del sistema dominante y en la relación de los pueblos indígenas con el Estado.

Autores como Fidel Tubino (2005b, s. f.) y Catherine Walsh $(2000,2009)$ proponen que una característica de la interculturalidad cuando parte desde los movimientos indígenas es que es crítica, ya que no busca incorporar los conocimientos indígenas a la matriz cultural dominante sino construir una propuesta política, epistémica y filosófica diferente de la hegemónica desde la perspectiva indígena. Es decir, cuando los movimientos indígenas incorporan en sus agendas el concepto de interculturalidad, este adquiere un significado propio, puesto que es implementado para lograr sus propios objetivos. Consideramos que esta resignificación constituye una interculturalidad crítica indígena y que se expresa en las experiencias realizadas en el Perú en educación superior intercultural por la Asociación Interétnica de Desarrollo de la Selva Peruana (Aidesep), tanto en la formación de maestros interculturales bilingües como en la de enfermeros técnicos en salud intercultural. Esta última será el foco de nuestro análisis. 
El concepto de interculturalidad desarrollado por Aidesep en la formación de jóvenes indígenas se construye en la práctica, en el hacer y en la reflexión que surge de esta acción práctica. Consideramos relevante reflexionar sobre esta interculturalidad indígena, ya que estas experiencias no parten de una noción de interculturalidad prestablecida sino que se trata de un concepto que se va construyendo. Esta idea de interculturalidad en construcción ha sido planteada por Chirif (2015) para repensar el concepto de territorio indígena. Chirif propone que dicho concepto es una noción que no responde a una definición única y homogénea, sino que es construida por cada pueblo indígena «desde su realidad específica actual, influida por una colonización de cinco siglos y que ha causado impactos diferentes» (2015).

En 2005 Aidesep inició el Proyecto de Formación de Enfermeros Técnicos en Salud Intercultural Amazónica (Pfetsia). Esta iniciativa se orientó al nivel tecnológico superior $y$, aunque para su funcionamiento se establecieron convenios interinstitucionales con institutos tecnológicos públicos (en tres provincias diferentes: Atalaya, Bagua y Nauta), la formación se desarrolló desde la perspectiva de Aidesep de manera autónoma.

Buscamos mostrar que la experiencia de formación del Programa de Salud Indígena de Aidesep (de aquí en adelante PSI-Aidesep) ha ido formando y reformulando progresivamente el concepto de interculturalidad en su implementación, orientada a fortalecer los sistemas de salud indígenas y mejorar la calidad de salud en la población (Hvalkof, 2003b). El Pfetsia se inició como un programa educativo de formación de promotores y luego dio pie al diseño de una propuesta novedosa de salud intercultural como carrera de nivel superior no universitaria. Esta experiencia, única en el país, sirvió de base para la elaboración de la carrera profesional de Enfermería Técnica Intercultural Bilingüe Amazónica ${ }^{1}$.

En este artículo hacemos un recorrido histórico del Pfetsia desde su concepción hasta la actualidad, con el fin de describir el proceso de resignificación de la interculturalidad en la formación en salud. Ponemos énfasis en las características de la propuesta curricular, así como en su relevancia política, pedagógica y epistémica, para la construcción de programas de formación en educación superior intercultural. A partir de documentos internos, publicaciones del Programa de Salud Indígena proporcionados por Aidesep (ver Anexo 1) y testimonios de

Esta carrera fue aprobada por silencio administrativo en 2015 (oficio 124-20015-MINEDU/ VMGP-DIGESUTPA basado en el expediente 0212184-2012). 
sus egresados, dirigentes y miembros del equipo técnico del PSI, analizamos la construcción e implementación de este concepto.

Los testimonios usados fueron recogidos en diferentes momentos: entre 2008 y 2011, durante la realización de la tesis doctoral de una de las autoras (Pesantes, 2014), y en el año 2016, en el proceso de sistematización del proyecto encargado por el Instituto de Estudios Peruanos y Aidesep (Cárdenas y Pesantes, 2016). A partir del análisis de estas fuentes, argumentamos que la salud intercultural para Aidesep es una propuesta en construcción que problematiza la interculturalidad en los niveles teórico y práctico. Cabe precisar que todos los autores han sido parte del PSI en diferentes roles.

\section{ANTECEDENTES: LAASOCIACIÓN INTERÉTNICA DE DESARROLLO DE LA SELVA PERUANA}

La Amazonía peruana es una región privilegiada por su diversidad natural, cultural y lingüística. En ella habitan cincuenta pueblos indígenas (http://bdpi.cultura. gob.pe/, 2017). Su historia se encuentra marcada por la exclusión del Estado, que ha vulnerado sus derechos y cuya presencia ha sido débil (García Hierro, Hvalkof y Gray, 1998; Santos Granero, 2004; Santos Granero y Barclay, 1999). A través de sus políticas, el Estado peruano ha tratado de asimilarlos al sistema dominante, extirpando e invisibilizando sus conocimientos, su forma de ver y de vivir, por considerarlas inferiores y subalternas a las prácticas occidentales (ibíd). En parte del imaginario nacional, los indígenas no son considerados ciudadanos con los mismos derechos que el resto de la población. Incluso un expresidente los describió como «ciudadanos de segunda categoría» (García Pérez, 2007). Para el expresidente García, los indígenas poseen ideologías obsoletas que deben ser superadas para no obstaculizar el crecimiento económico del país (2007). Esto ha sucedido a lo largo de la vida republicana, como cuando se decidió la construcción de la Carretera Marginal del presidente Belaunde Terry en la década de 1960.

A pesar de esta historia de asimilación, de inclusión forzada a través de la castellanización y occidentalización, los pueblos indígenas mantienen sus conocimientos, sus propias formas de percibir el mundo y de vivir en él. Es desde esta visión indígena que se establecen diálogos o se confrontan los conocimientos occidentales. También es desde allí que se formulan propuestas de políticas, de intervenciones y de acciones orientadas a responder a la desatención histórica que sufren los pueblos indígenas y a la vulneración de sus derechos. 
Para conseguir el respeto de sus derechos y la defensa de sus territorios, que es la base de la supervivencia, los pueblos indígenas peruanos se organizaron y en 1980 se funda Aidesep, principal organización indígena de la Amazonía peruana (Inoach Shawit y Reátegui Silva, 2003). En la actualidad, Aidesep representa a 1809 comunidades de toda la Amazonía, afiliadas a 65 federaciones de base y nueve organismos descentralizados (www.aidesep.org). Aidesep cuenta con nueve programas nacionales: Bosques, Pueblos Indígenas en Situación de Aislamiento y Contacto Inicial (Piaci), Centro de Información y Planificación Territorial de Aidesep (Cipta), Comunicación, Educación, Economía, Hidrocarburos, Mujer y Salud (www.aidesep.org).

\section{EL PROGRAMA DE SALUD INDÍGENA DE AIDESEP}

El Programa de Salud Indígena (PSI) se creó en 1991 con la finalidad de desarrollar los sistemas de salud indígenas y de mejorar la calidad de la salud de las comunidades, implementando actividades y proyectos centrados en fortalecer y desarrollar los sistemas de salud indígenas y la atención de las enfermedades prevalentes en la Amazonía ${ }^{2}$ (Reátegui, 2005). En un inicio, la estrategia consistió en desplegar equipos itinerantes en comunidades indígenas de la región Ucayali, conformados por una enfermera, un representante de la organización y un especialista de la medicina indígena, como parteras, vaporadoras o sobadores (Aidesep, 2010, 2011; Hvalkof, 2003a; Reátegui, 2005; Reátegui Silva, 2003).

En el marco de esta experiencia, el PSI-Aidesep desarrolló un modelo de diálogo intercultural entre personal de salud y especialistas indígenas y de capacitación de promotores de salud, sentando las bases de una "propuesta para una educación institucionalizada del promotor de salud» (Hvalkof, 2003a, p. 9). Como recuerda Juan Reátegui, exdirector del PSI: «Trabajamos largamente con el desarrollo de los sistemas de salud indígena, y eso permitió que, después de todo ese recorrido que hicimos [...], pudiéramos fijar una metodología y una estrategia de formación de recursos humanos» (2008, entrevista personal). Vemos que la formación de profesionales de salud intercultural altamente calificados era una prioridad del PSI-Aidesep.

Para Aidesep, un profesional altamente calificado es aquel que conoce los territorios indígenas, realiza sus atenciones incorporando elementos de los siste-

2 Este proyecto contó con el apoyo de la agencia danesa Nordeco y la Fundación Karen Elisse Jensen. 
mas médicos indígenas, respeta sus prácticas sanitarias y trabaja coordinadamente con los especialistas indígenas (Pesantes, 2014). Es decir, un profesional que sea un puente entre las medicinas indígenas y la medicina occidental, con una formación que responda a las necesidades en salud de los pueblos indígenas (Cárdenas y Pesantes, 2017). La apuesta por formar un profesional con habilidades interculturales es una respuesta a la poca pertinencia del personal de salud que suele ir a trabajar a las comunidades indígenas. Estos profesionales, usualmente mestizos, han sido formados bajo un enfoque biomédico hegemónico quien no está preparado para atender a población indígena. Como expresa Reátegui:

Existe una brecha [entre la población indígena y el personal de salud]; los enfermeros [mestizos] no hablan el idioma, no saben comer lo que come la gente, nunca han andado en bote, en las trochas; ese tipo de gente ¿cómo puede ayudar a un mundo diverso, que tiene otro contexto, otra forma de pensar y de vida? (2008, entrevista personal)

Inicialmente, el PSI-Aidesep quería preparar jóvenes indígenas como promotores (Reátegui Silva, 2003). Sin embargo, hubo factores que evidenciaron la necesidad de formarlos como técnicos en salud intercultural. Por ejemplo, se identificó que los promotores indígenas no podían manejar la «incomunicación entre las sabidurías ancestrales de los pueblos y la medicina llamada "científica"» (Rodríguez Torres, Valdez y Reátegui Silva, 2009, p. 90), y al no estar insertados laboralmente en el sistema de salud estatal, dependían del financiamiento y la asistencia técnica de Aidesep para cumplir sus funciones. Cuando los proyectos concluían, retornaban a sus actividades cotidianas.

Otro factor que contribuyó a consolidar la necesidad de profesionalizar indígenas en salud fue el respaldo de las autoridades locales del sector educación en Atalaya, el primer lugar donde se formaron los Técnicos en Enfermería Intercultural Amazónica (Tesia). Ellos fueron formados con la expectativa de contar con el reconocimiento del Estado y para insertarse laboralmente dentro del Minsa.

En la transición de la capacitación de promotores a la formación de Tesia, el equipo del PSI-Aidesep percibió que su propuesta se orientaba bajo un enfoque intercultural (Reátegui, 2005). El perfil profesional propuesto buscaba «recuperar, desarrollar y proteger los conocimientos y tecnologías de salud indígenas; incorporar el enfoque intercultural en los sistemas de salud del Estado peruano; y, contar con recursos humanos indígenas formados en una visión intercultural de la salud» (Rodríguez Torres et al., 2009, p. 92). Es decir, se esperaba que, a través de una formación intercultural desde la visión indígena, estos profesionales 
promuevan la implementación de un subsistema de salud intercultural al interior del sistema de salud estatal.

La propuesta de formación de Tesia implicó diseñar e implementar un currículo intercultural por competencias profesionales desde la perspectiva indígena. La propuesta pedagógica en educación superior intercultural de Aidesep se caracteriza por ser flexible y estar en permanente construcción, buscando responder a las necesidades y demandas en salud de los pueblos indígenas (Cárdenas y Pesantes, 2017). Así, su implementación se alinea con la política y los intereses de Aidesep, que buscan el reconocimiento de los sistemas indígenas de salud.

Después de tres experiencias de formación desarrolladas entre 2005 y 2013, la propuesta de formación de técnicos en enfermería de Aidesep ha servido como base para la creación de la Carrera de Enfermería Técnica en Salud Intercultural Amazónica. Para su creación, esta carrera fue presentada ante el Ministerio de Educación (Minedu) por el instituto Joaquín Reátegui de Nauta con el apoyo técnico de Aidesep. La carrera fue aprobada por el Minedu en 2015 por silencio administrativo. Esta figura legal es controversial, ya que, para los dirigentes indígenas, es una forma de no querer reconocer el aporte de las organizaciones a la formulación de las políticas públicas para pueblos nativos. Por otro lado, se puede interpretar como una discriminación hacia las capacidades técnicas de los indígenas para formular propuestas sólidas.

En el I Encuentro de Enfermeros Técnicos en Salud Intercultural de Aidesep de $2016^{3}$, dirigentes, estudiantes y equipo técnico llegaron a la conclusión de que es importante que la organización indígena participe directamente en la formación de los futuros enfermeros técnicos en salud intercultural. Solo la participación de Aidesep garantizará que, en nombre de la interculturalidad, no se subestimen los conocimientos indígenas y se sigan formando profesionales en salud desde la perspectiva indígena. En ese sentido, se percibe como riesgoso que el instituto Joaquín Reátegui de Nauta actualmente ofrezca la carrera diseñada por Aidesep sin convocarlo a que brinde la asesoría respectiva para el enfoque indígena e intercultural. No tener a Aidesep con un rol de acompañamiento o asesoría puede traer como consecuencia que el enfoque intercultural usado, lejos de ser crítico, se vuelva funcional al Estado. Es decir, que no cuestione el paradigma occidental del sistema de salud oficial que no siempre es pertinente para trabajar con la población indígena.

Realizado en Lima los días 28, 29 y 30 de mayo de 2016 y financiado por el Instituto de Estudios Peruanos. 
Durante ese encuentro, los participantes concluyeron, además, que para crear un subsistema de salud intercultural, es necesario que los enfermeros técnicos formados por Aidesep se conviertan en licenciados en enfermería. Esto les permitiría ocupar cargos de mayor jerarquía dentro del Minsa, y así, participar en la formulación e implementación de políticas, normas y protocolos que consideren la mirada indígena.

\section{FORMANDO A LA ÉLITE INDÍGENA EN SALUD INTERCULTURAL}

Yo no entendía bien qué era eso de interculturalidad. Asistimos a un taller de UNICEF sobre pertinencia intercultural en Nieva. Ahí estaban el personal de salud y las parteras, pero no se entendían. Ahí me di cuenta de que para eso servía la interculturalidad.

Mis compañeros y yo entendíamos al personal y a la partera y podíamos explicar los conocimientos de ambos lados, pero también explicar por qué es importante la medicina indígena y por qué se debe trabajar con los sabios indígenas porque ellos conocen cosas que el personal de salud no, y son conocimientos buenos

(Nevin Chumpik, egresado de Bagua 2011, entrevista personal)

Para Aidesep, el enfermero técnico en salud intercultural es un profesional capaz de articular la perspectiva occidental y la perspectiva indígena de la salud de manera creativa y en función de las necesidades de los pueblos indígenas. Es decir, Aidesep pensó en este profesional como una especie de puente, de bisagra que puede transitar entre los dos sistemas médicos, generando espacios para que ambos conocimientos puedan encontrarse y articularse en la atención ofrecida en los establecimientos de salud. Como menciona Alberto Pizango, expresidente de Aidesep:

Nosotros queremos que nuestros jóvenes sean los responsables de implementar la política indígena de salud, que sean nuestros interlocutores directos con el Estado, que se conviertan en nuestros asesores, pero que también trabajen en el Estado y desde ahí promuevan cambios en el sistema de salud, que cuente con políticas donde los conocimientos médicos en salud estén reconocidos como válidos, como los de ellos (2009, conversación personal).

El Pfetsia se desarrolló durante el período 2005-2015 en tres zonas de la Amazonía peruana: Atalaya en la zona este, Bagua en la zona nororiental y Nauta en el norte del país, y hasta el momento tiene 69 egresados. Una de las características de estos proyectos es que fueron itinerantes, se desarrollaron en 
diferentes lugares y procuraron que los pueblos indígenas ${ }^{4}$ a los que pertenecían los estudiantes fueran de la misma familia lingüística y estuvieran afiliados a las federaciones de base de Aidesep (Cárdenas y Pesantes, 2017). Así, en Atalaya participaron jóvenes yines, shipibos, asháninkas y ashéninkas de las federaciones de OAGP, OIRA y Ordeconadit; en Bagua participaron awajun, wampis y kechwas de la regional Orpian-P, y en Nauta Achuars de Feconaco.

Implementar esta propuesta no fue una tarea fácil, como se observa en el comentario inicial, dado por un egresado del programa. Lograr que los jóvenes indígenas se apropien y pongan en práctica la salud intercultural implicó una serie de retos que las organizaciones indígenas tendrán que ir solucionando en el camino. Uno de los principales desafíos enfrentados fue el no reconocimiento del Ministerio de Educación (Minedu) de la mención en salud intercultural al título de enfermeros técnicos en salud. Los jóvenes estudiantes tenían muchas expectativas de tener este título.

Para conseguir el reconocimiento oficial de su propuesta, Aidesep uso como sustento jurídico la Ley General de Educación 28044 (2004). Esta ley promovía la Educación Comunitaria, permitiendo a la sociedad complementar la formación de los profesionales para adecuarlos a la realidad local de la zona (Rodríguez Torres et al., 2009). Esto permitió la implementación de un modelo de cogestión, entre los institutos tecnológicos y las organizaciones de base de Aidesep, con el fin de desarrollar una innovación pedagógica a la carrera de enfermería técnica, y posteriormente, solicitar al Minedu que les otorgue la mención en salud intercultural al título profesional.

A través de la firma de convenios con los institutos tecnológicos, Aidesep garantizó que el PSI se encargase de conducir la innovación pedagógica y los institutos fueran los responsables de supervisar su desarrollo (Aidesep, 2005b, 2009, 2012). Este modelo de cogestión autónoma dentro de los institutos permitió que los proyectos de formación funcionen paralelamente bajo su propio modelo pedagógico, escojan su propia plana docente — que incluía personal médico y especialistas indígenas de amplia trayectoria recomendados por las propias organizaciones-, así como un horario que incluía clases tanto en la mañana como en la tarde.

Como se observa en los Proyectos Educativos Institucionales de las tres experiencias de formación (Aidesep, 2005a, 2009, 2012), estas fueron conducidas por una directora encargada de la gestión y ejecución directa del proyecto, de

$4 \quad$ El Ministerio de Cultura de Perú reconoce que en este país viven 55 grupos etnolingüísticos. 
las coordinaciones con las autoridades del instituto y de las capacitaciones a los profesores - tanto del instituto como del proyecto-, con la finalidad de asegurar una formación intercultural desde la perspectiva indígena. Es interesante resaltar que la labor de la directora, del equipo técnico y de los estudiantes fue supervisada por el Consejo Asesor Indígena y por los dirigentes regionales y nacionales, quienes tenían reuniones periódicas con los responsables de la ejecución del proyecto para supervisar su desarrollo.

La autonomía de Aidesep para desarrollar la formación de enfermeros técnicos en salud intercultural fue posible debido a que Aidesep contaba con financiamiento exterior para la ejecución de sus proyectos. Creemos que, sin este financiamiento, un modelo de esta naturaleza no hubiera sido posible, ya que el sistema educativo estatal peruano aún no está preparado para implementar un modelo de cogestión con las organizaciones indígenas para la formación de recursos humanos.

La propuesta curricular y de formación se construyó tomando como columna vertebral los conocimientos indígenas, los cuales no se encuentran en los libros ni en las bibliotecas sino en la memoria de los sabios indígenas que durante años han ido acumulándolos a través de la experimentación, del contacto y el estudio de las plantas medicinales. Esta propuesta busca que los profesionales formados bajo un enfoque monocultural entiendan que el conocimiento biomédico occidental es uno más (no el único válido) con aportes y límites, como el conocimiento indígena, y que necesitan trabajar de la mano con ellos.

Como mencionó Fermín Murayari (2011), uno de los principales sabios indígenas involucrado en la experiencia, «este no es un proyecto para que los médicos, usando nuestros conocimientos, nos pongan los suyos: es para que los jóvenes indígenas fortalezcan nuestra medicina, la respeten y respeten a los sabios indígenas» (conversación personal). Este testimonio del maestro Murayari, así como la cita con la que damos inicio esta sección, muestran cómo Aidesep fue construyendo en la práctica su modelo de educación superior tecnológica intercultural. Además, vemos cómo su propuesta de formación implicó un doble desafío: por un lado, interculturalizar el currículo, y por otro, desarrollar su propuesta de salud intercultural indígena.

El modelo pedagógico curricular desarrollado por Aidesep es pertinente a la realidad amazónica, porque su construcción tuvo como referentes las características de los pueblos indígenas, no solo en su aspecto cultural sino también en cuanto a su realidad socioeconómica. En la sistematización de la experiencia pedagógica de Aidesep (2017), resaltamos que uno de los aspectos relevantes de sus proyectos es su adaptabilidad a las características socioculturales de 
cada zona; es decir que, para cada nueva experiencia, el perfil del egresado fue modificándose y adaptándose según el nivel de escolaridad de los estudiantes y la realidad de los pueblos indígenas de la zona.

Adicionalmente, los proyectos de formación dieron mucha importancia al programa propedéutico que fue transversal a los tres años de formación, pues los jóvenes llegaban con deficiencias en sus capacidades instrumentales en las áreas de lenguaje y matemática producto de la mala calidad de la educación básica regular ofrecida por el Estado en sus comunidades de origen (Rodríguez Torres et al., 2009). Además del programa propedéutico, hubo una tutoría indígena y académica permanente que acompañó a estos jóvenes tanto en los aspectos volitivos y académicos como en la supervisión, monitoreo, capacitación y acompañamiento pedagógico de los docentes, para que consiguieran aproximarse a las formas de aprendizaje indígena y reflejarlas en sus procesos de enseñanza-aprendizaje (Cárdenas y Pesantes, 2017).

En este adaptar y modificar su propuesta, Aidesep consiguió diseñar el perfil profesional no solo de un enfermero técnico sino de un licenciado en enfermería en salud intercultural, que es lo que se necesita en el sector salud, pues en las comunidades alejadas se requiere profesionales con capacidad de resolver múltiples problemas de salud y no tener que derivar al paciente a establecimientos con mayor capacidad resolutiva ubicados a horas o días de distancia. Por lo general, el personal mestizo está formado para el trabajo en contextos urbanos muy diferentes de los de las comunidades indígenas.

El Pfetsia no solo tenía objetivos pedagógicos, sino también políticos, en tanto que buscaba insertar a sus egresados en el sistema de salud estatal y, una vez dentro, aspiraba a que estos profesionales pudiesen promover el desarrollo de un subsistema de salud intercultural. Este subsistema implica que exista un modelo de atención específico para población indígena que parta de una perspectiva indígena del proceso salud-enfermedad-curación. La aspiración política de Aidesep es que dentro del Minsa se cree una instancia en salud intercultural de carácter ministerial. Para ello, es necesario formar a la élite indígena en salud encargada de formular estas propuestas y ser sus interlocutores directos con el Estado.

Para garantizar que estos jóvenes se conviertan en los interlocutores del movimiento indígena, una premisa importante en la formación fue el fortalecimiento de la identidad indígena. Como señala Alberto Pizango (2009) — expresidente de Aidesep - en una conversación personal: «los estudiantes del programa debían egresar más indígenas de lo que ingresaron». 
Es por ello que el Pfetsia promovió la valoración positiva de las identidades indígenas de sus estudiantes. Sin embargo, al inicio de las experiencias formativas se observó que estas se encontraban totalmente debilitadas como consecuencia del proceso de occidentalización y de pérdida de algunos valores y conocimientos indígenas promovido por una escolarización sin enfoque intercultural.

Los primeros meses hubo una resistencia muy activa frente al discurso indígena (o indigenista) del proyecto. Incluso se negaron a usar sus indumentarias indígenas para las actividades sociales del proyecto y a participar en las ceremonias del ayahuasca ${ }^{5}$. [...] Sin embargo, después de algunos meses de trabajo de los tutores indígenas y del conjunto del personal del proyecto, la mayoría de los chicos se fueron acercando cada vez más a sus identidades étnicas (Rodríguez Torres et al., 2009, p. 103).

La importancia del fortalecimiento de las identidades indígenas en la propuesta de formación partió de la premisa de que los estudiantes percibían la sociedad occidental como superior a los pueblos indígenas. Dicha idea fue trabajada a través de la revaloración de los conocimientos y las tecnologías indígenas, que fueron presentadas en un mismo nivel valorativo que los conocimientos occidentales. Como expresa uno de los egresados del programa:

A mí no me gustaba usar cushma $a^{6}$ La directora decía, ¿dónde está tu cus$h m a$ ? La escondía para que ella crea que no tenía. El día que nos dieron el título, estaba bien contento, quería usar mi cushmita, me sentía orgulloso de ser asháninka y no sentía vergüenza (Eduardo Rengifo, egresado de Atalaya, 2008, entrevista personal).

La formación de enfermeros técnicos en salud intercultural plantea una interculturalidad desde la visión indígena que parte del reconocimiento de los sistemas de salud indígenas, los cuales se consideran tan valiosos y efectivos como la medicina occidental. Utilizar la medicina occidental no significa que los indígenas hayan abandonado sus propias formas de interpretar y resolver los problemas de salud (Reátegui, 2008, entrevista personal), sino que como producto del contacto con los no indígenas han aparecido enfermedades para las cuales necesitan incorporar tratamientos de la medicina occidental.

5 Ayahuasca es una bebida alucinógena hecha del tronco de la vid ayahuasca Banisteriopsis caapi. Rara vez se consume sola; casi siempre se le agregan otras plantas (Beyer, 2009). La consumen sobre todo los chamanes, pero cada pueblo indígena tiene rituales y usos diferentes.

6 Vestimenta tradicional indígena usada por varones y mujeres. Cada grupo indígena amazónico usa una cushma de material diferente o con decoraciones diferentes. 
La propuesta de interculturalidad en salud desarrollada por Aidesep entiende la medicina occidental como una alternativa más de atención. Esta idea se observa en el testimonio de Henderson Rengifo - expresidente de Aidesep- cuando dice que «la búsqueda de cura para los problemas y necesidades en salud que presentamos ya no se realiza de forma exclusiva en nuestros sistemas, por lo contrario, hacemos uso tanto de las medicinas indígenas como de la oferta en salud ofrecida por el Estado peruano» (Chávez et al., 2015, p. 15). La interculturalidad en salud que propone Aidesep parte de una reflexión de la historia de los pueblos indígenas y de las necesidades concretas en salud que enfrenta está población.

Algunos elementos claves de la puesta en práctica de la interculturalidad en el Pfetsia son:

- Los conocimientos occidentales fueron reflexionados e incorporados a una estructura que es pensada desde la lógica indígena.

- El diseño de un nuevo perfil de enfermero técnico pensado para atender a comunidades indígenas.

- La participación garantizada de las organizaciones de base a través de la conformación de Consejos Asesores Indígenas, quienes fueron la instancia máxima de decisión de las acciones desarrolladas en el proyecto.

- Los cursos del área indígena fueron dictados por sabios indígenas, quienes no contaban con un título universitario, pues dentro de la perspectiva de Aidesep el curandero, la partera o cualquier especialista de la medicina indígena debe recibir el reconocimiento de sus conocimientos y de sus prácticas en las comunidades. La premisa de su contratación se sustenta en que sus conocimientos son validados por el sistema de conocimiento indígena y no occidental.

- La autonomía para conducir sus propias experiencias impidió que sesgos relacionados con la especialización occidental sean una barrera que impida su inserción como docentes, ya que no se duda del conocimiento que tienen debido a que este es considerado válido desde la perspectiva indígena.

\section{DE QUÉ INTERCULTURALIDAD NOS ESTÁ HABLANDO AIDESEP}

En algunos documentos que abordan la experiencia de enfermeros técnicos en salud intercultural (Cárdenas y Pesantes 2017; Chavez et al., 2015; Rodríguez Torres et al., 2009), se señala que Aidesep plantea la interculturalidad como una propuesta para producir cambios en el sistema hegemónico de salud. Si bien el Minsa cuenta con normativas para la incorporación de la interculturalidad como 
un enfoque transversal en su atención (Minsa, 2006a, 2006b), esta no se ha logrado implementar de forma que mejore la calidad de atención de los pueblos indígenas. En aquellos pocos ejemplos en los que el Minsa ha logrado usar un enfoque intercultural, como en la atención vertical del parto, la propuesta ha cumplido un papel funcional al sistema hegemónico dominante (Guerra-Reyes, 2009).

Aidesep empieza a construir la interculturalidad en salud a partir de sus experiencias en la formación intercultural de indígenas como enfermeros técnicos, y en el poner en práctica su noción de interculturalidad observamos que se da de una manera crítica, pues ha adquirido «una significación político-normativa más compleja (Tubino, 2005), ya que busca promover cambios en las estructuras del sistema político, epistémico, cognitivo y filosófico dominante (Walsh, 2009). Así, la interculturalidad aparece como un proyecto político decolonizador relacionado con la lucha de los pueblos indígenas por construir un proyecto que transforme las estructuras y cree nuevas formas de poder en el área de salud.

La interculturalidad crítica, según Marisol de la Cadena, es algo por construirse y «no es un proceso fluido, mucho menos automáticamente exitoso» (2006, p. 219). Es en esta línea en la que debemos ver la noción en salud intercultural de Aidesep como una propuesta en proceso de consolidación.

Una de las características de la interculturalidad en salud propuesta por Aidesep es que se produce un quiebre en el lugar que ocupa el proponente del diálogo intercultural, pasando de una visión etnocéntrica que parte de un «yo» occidental y un «otro» indígena a una relación donde el «otro» pasa a ser el sujeto occidental con quien se busca la comunicación horizontal para la construcción de una propuesta de salud intercultural. Así, la organización indígena se vuelve un sujeto activo y proponente del proceso intercultural (Rodríguez Torres et al., 2009). Partiendo de una visión indígena de la interculturalidad, Aidesep desarrolla sus experiencias y las pone en práctica, para desde ese hacer, reflexionar teóricamente a posteriori.

\section{REFLEXIONES FINALES}

Hemos descrito cómo la interculturalidad que orienta la formación en salud intercultural parte desde la visión indígena. Esta visión se caracteriza por ser una propuesta en construcción que, si bien dialoga con algunas referencias teóricas, se apropia y resignifica la idea de diálogo sobre la base del respeto, donde los proponentes de este diálogo son los indígenas, que ven en la incorporación de la interculturalidad en su agenda la posibilidad de responder a los nuevos 
contextos que influyen en sus dinámicas internas, marcados por quinientos años de colonialidad.

Consideramos que la idea de interculturalidad desarrollada por Aidesep se basa en el reconocimiento y la validez de los sistemas médicos de salud indígenas, así como el uso del sistema occidental para determinadas situaciones.

En este escenario, donde los indígenas no renuncian al desarrollo de sus sistemas de salud, se hace necesario que planteen al Estado el desarrollo de un sistema de salud que reconozca sus conocimientos y prácticas sanitarias. Es así que la formación en salud intercultural aparece como una respuesta a este nuevo contexto, que demanda la formación de profesionales capaces de dialogar con los dos conocimientos y las diferentes perspectivas del mundo.

Aidesep también propone una educación superior intercultural, a través de la cual se prepare a profesionales capaces de transformar los servicios de salud del Estado desde abajo (y no a través de mandatos del gobierno) con nuevas maneras de concebir los servicios de salud, de tal manera que respondan a las demandas de las organizaciones indígenas. Un subsistema que parta de un cuestionamiento de los procesos históricos que relegan los conocimientos médicos indígenas por no ser «científicamente» comprobados. Esto implica que hay una posición contestataria frente al monopolio que tiene la biomedicina occidental para establecer los mecanismos de validación de conocimientos.

La propuesta de interculturalidad en salud desarrollada por Aidesep solo podrá implementarse si se garantiza su autonomía para tomar decisiones. Esto es posible cuando la organización consigue el financiamiento que le permite conducir directamente sus experiencias de formación, porque son percibidas como suyas y responden a su agenda política, ya que le permiten intentar producir transformaciones en el sistema y no ser incorporada a ellas a través del uso de una interculturalidad funcional al sistema hegemónico.

Revisando algunas anotaciones sobre el trabajo dentro del PSI de Aidesep, identificamos que existe una apropiación y resignificación del concepto intercultural desde la mirada de Aidesep. Reconocer este aspecto es importante, porque resalta el lugar epistémico desde el cual está siendo planteada la interculturalidad.

Por lo propuesto, y tomando como base el concepto de interculturalidad crítica de los autores Tubino y Walsh, planteamos la idea de una interculturalidad indígena crítica. Esta idea cuestiona una interculturalidad crítica estatal, así como los discursos que asumen una propuesta de interculturalidad crítica, pero al momento de su implementación son ejecutados dentro de los dominios del estado hegemónico y responden a las estructuras estatales. 
Lo que Aidesep nos está enseñando con su propuesta de formación de enfermeros técnicos en salud intercultural es que es posible que la interculturalidad crítica pase del discurso a la práctica y que, a pesar de sus contradicciones y complejidades, empiece no solo a enunciarse o pensarse, sino sobre todo a hacerse. Esto solo será posible si los indígenas participan en la construcción de propuestas interculturales, no solo para validar modelos desarrollados por el Estado, sino también para desarrollar autónomamente sus propias propuestas de interculturalidad en diferentes ámbitos.

\section{REFERENCIAS BIBLIOGRÁFICAS}

Aidesep (2005a). Ayuda Memoria del Programa de Salud Indígena. Lima: Asociación Interétnica de Desarrollo de la Selva Peruana.

Aidesep (2005b). Proyecto Educativo Institucional de Atalaya. Atalaya: Asociación Interétnica de la Selva Peruana.

Aidesep (2009). Proyecto Educativo Institucional de Bagua. Bagua: Asociación Interétnica de Desarrollo de la Selva Peruana.

Aidesep (2010). De médicos y sheripiaris: Proyecto Piloto de Salud Intercultural. Atalaya: Masato Producciones.

Aidesep (2011). Informe final. Proyecto de formación de enfermeros técnicos en salud intercultual amazónica para el fortalecimiento de los sistemas de salud indígena de los pueblos jíbaros de la Amazonía Norte del Perú (Bagua). Bagua: Asociación Interétnica de Desarrollo de la Selva Peruana.

Aidesep (2012). Evaluación final. Formación de enfermeros técnicos en salud intercultural amazónica para el fortalecimiento de los sistemas de salud indígena de los pueblos jibaros de la Amazonía Norte del Perú.

Cárdenas Palacios, Cynthia y Amalia Pesantes (2017). Entrecruzando rios: sistematización de la propuesta pedagógica de formación de enfermeros técnicos en salud intercultural de Aidesep. Lima: Instituto de Estudios Peruanos y Asociación Interétnica de Desarrollo de la Selva Peruana.

Chávez, C., C. Yon y C. Cárdenas (2015). El aporte de los egresados del Programa de Formación de Enfermeros Técnicos en Salud Intercultural Amazónica de Aidesep a la Salud Intercultural: estudio de caso en dos comunidades de Amazonas. Lima: Instituto de Estudios Peruanos.

Chirif, A. (2015). Territorios indigenas en la coyuntura actual. Paper presented at the Políticas de desarrollo, territorio y consulta previa. 
De la Cadena, M. (2006). The Production of Other Knowledges and its Tensions: From Andeanist Anthropology to Interculturalidad. En G. Lins-Ribeiro y A. Escobar (eds.), World Anthropologies: Disciplinary Transformation within Systems of Power (pp. 201-224). Nueva York y Oxford: Berg Publishers.

García Hierro, P., S. Hvalkof y A. Gray (1998). Liberation through land rights in the Peruvian Amazon (Vol. 90). Copenhagen: IWGIA.

García Pérez, A. (2007). El síndrome del perro del hortelano. El Comercio.

Guerra-Reyes, L. (2009). Implementing an Intercultural Birth Care Policy: The role of indigenous identity in the Peruvian manternal care. Anthropology News, 50(3), 13-14. https://doi.org/10.11144/Javeriana.UH80.smai

http://bdpi.cultura.gob.pe/ (2017). Base de datos de pueblos indígenas u originarios. Recuperado el 23 de junio de 2017.

Hvalkof, S. (2003a). Lugar, población e historia. En S. Hvalkof (ed.), Sueños amazónicos: un programa de salud indigena en la selva peruana (pp. 36-87). Copenhague: Fundación Karen Elise Jensen y Nordeco.

Hvalkof, S. (ed.). (2003b). Sueños amazónicos: un Programa de Salud Indígena en la Selva Peruana. Copenhagen: Nordeco.

Inoach Shawit, G. y J. Reátegui Silva (2003). Aidesep y la perspectiva indígena. En S. Hvalkof (ed.), Sueños amazónicos... un programa de salud indígena en la selva peruana (pp. 16-27). Copenhague: Fundación Karen Elise Jensens y Nordeco.

López, L.E. (2001). La cuestión de la interculturalidad y la educación latinoamericana. Paper presented at the Séptima Reunión del Comité Regional Intergubernamental del Proyecto Principal de Educación en América Latina y el Caribe.

Minsa (2006a). Documento técnico: marco conceptual de los derechos humanos, equidad de género e interculturalidad en salud. Lima: Ministerio de Salud del Perú.

Minsa (2006b).Norma técnica de salud para la transversalización de los enfoques: derechos humanos, equidad de género e interculturalidad en salud, 014-2002-SA C.F.R.

Reátegui Silva, J. (2003). El origen del Programa de Salud Indígena: entrevista con el director del programa. En S. Hvalkof (ed.), Sueños amazónicos... un programa de salud indígena en la selva peruana (pp. 98-104). Copenhague: Fundación Karen Elise Jensen y Nordeco.

Reátegui, J. (2005). Las políticas de salud de los pueblos indígenas de la Amazonía peruana. Lima: Aidesep.

Reátegui, J. (2008). Entrevista a Juan Reátegui, director técnico del Programa de Salud Indígena de Aidesep. En M.A. Pesantes (ed.). Lima. 
Rodríguez Torres, A., M.I. Valdez Felipe y J. Reátegui Silva (2009). Formación de Enfermeros Técnicos en Salud Intercultural: una experiencia de cooperación entre las organizaciones indígenas de la Amazonía peruana, el Estado y un instituto tecnológico. En D. Mato (ed.), Educación superior, colaboración intercultural y desarrollo sostenible/ buen vivir - Experiencias en América Latina (pp. 87-114). Caracas: Instituto Internacional de la Unesco para la Educación Superior en América Latina y el Caribe (IESALC-Unesco).

Santos Granero, F. (2004). Selva Central. History, Economy, and Land Use in Peruvian Amazonia. Washington D.C.: Smithsonian.

Santos Granero, F. y F. Barclay (1999). Tamed Frontiers: Economy, Society and Civil Rights in Upper Amazonia. Boulder: Westview.

Tubino, F. (2005a). La interculturalidad crítica como proyecto ético-político. Paper presented at the Encuentro Continental de Educadores Agustinos. Retrieved from http://oala.villanova.edu/congresos/educacion/lima-ponen-02.html

Tubino, F. (2005b). La praxis de la interculturalidad en los estados nacionales latinoamericanos. Cuadernos Interculturales, 3(005), 83-96.

Tubino, F. (s. f.). Del interculturalismo funcional al interculturalismo crítico. Recuperado 12 de junio de 2017, de http://red.pucp.edu.pe/ridei/libros/del-interculturalismofuncional-al-interculturalismo-critico/

Walsh, C. (2000). Propuesta para el tratamiento de la interculturalidad en la educación. Lima.

Walsh, C. (2009). Interculturalidad crítica y pedagogía de-colonial: Apuestas (des)de el in-surgir, re-existir y re-vivir. Paper presented at the seminario Interculturalidad y Educación Intercultural. 